\title{
Non-commuting two-local Hamiltonians for quantum error suppression
}

\author{
Zhang Jiang ${ }^{1,2, *}$ and Eleanor G. Rieffel ${ }^{1, \dagger}$ \\ ${ }^{1}$ Quantum Artificial Intelligence Laboratory (QuAIL), \\ NASA Ames Research Center, Moffett Field, California 94035, USA \\ ${ }^{2}$ Stinger Ghaffarian Technologies Inc., \\ 7701 Greenbelt Rd., Suite 400, Greenbelt, MD 20770
}

(Dated: September 10, 2018)

\begin{abstract}
Physical constraints make it challenging to implement and control many-body interactions. For this reason, designing quantum information processes with Hamiltonians consisting of only one- and two-local terms is a worthwhile challenge. Enabling error suppression with two-local Hamiltonians is particularly challenging. A no-go theorem of Marvian and Lidar (Phys Rev Lett 113(26):260504, 2014) [1] demonstrates that, even allowing particles with high Hilbert-space dimension, it is impossible to protect quantum information from single-site errors by encoding in the ground subspace of any Hamiltonian containing only commuting two-local terms. Here, we get around this no-go result by encoding in the ground subspace of a Hamiltonian consisting of non-commuting two-local terms arising from the gauge operators of a subsystem code. Specifically, we show how to protect stored quantum information against single-qubit errors using a Hamiltonian consisting of sums of the gauge generators from Bacon-Shor codes (Bacon in Phys Rev A 73(1):012340, 2006) [2] generalized-Bacon-Shor code (Bravyi in Phys Rev A 83(1):012320, 2011) [3]. Our results imply that non-commuting two-local Hamiltonians have more error-suppressing power than commuting two-local Hamiltonians. While far from providing full fault tolerance, this approach improves the robustness achievable in near-term implementable quantum storage and adiabatic quantum computations, reducing the number of higher-order terms required to encode commonly used adiabatic Hamiltonians such as the Ising Hamiltonians common in adiabatic quantum optimization and quantum annealing.
\end{abstract}

doi:10.1007/s11128-017-1527-9

PACS numbers: 03.67.Pp, 03.65.Ud, 03.67.Lx

Keywords: Error suppression; Subsystem quantum error-correcting code; Two-local Hamiltonian; Bacon-Shor code; adiabatic quantum computation; quantum annealing.

\footnotetext{
* zhang.jiang@nasa.gov

† eleanor.rieffel@nasa.gov
} 


\section{INTRODUCTION}

In order to realize the benefits of quantum computation $[4,5]$, machines capable of robust quantum computation must be built. An active area of research explores what resources are needed to achieve physically implementable robust quantum computing. While the ultimate goal is to achieve a fully fault-tolerant implementation, it is also important to determine what resources enable more robust, if not fully fault-tolerant, quantum computations, so as to enable a richer set of experiments to be performed on hardware implementable in the near term.

One important question is to determine when many-body interactions are required and when two-local interactions suffice. Even determining the resources required to support the robust storage of quantum information is challenging [6]. Two-local interactions have been realized in all leading candidates for near-term quantum computation, while three-local and higher locality interactions remain an implementation challenge. Within adiabatic quantum computing, a common approach to increase robustness is error suppression through energy gap protection (EGP) $[7,8]$ in which quantum information is encoded in the ground subspace of a Hamiltonian derived from an error-detecting code and an error-suppressing term is added to the total Hamiltonian, penalizing states outside that subspace.

Unfortunately, any error-suppressing Hamiltonian constructed from a stabilizer code must contain interactions involving three or more qubits to protect against single-qubit errors. In [1], Marvian and Lidar proved a stronger negative result, namely that energy gap protection using an error suppressing Hamiltonian that is the sum of two-local commuting terms cannot suppress single-site errors even with particles of arbitrarily large Hilbert space dimension. Their proof uses a powerful theorem due to Bravyi and Vyalyi [9], generalized by Aharonov and Eldar [10], which says that ground states of commuting two-local Hamiltonians can have only short-range two-body entanglement. This no-go result implies that, in the words of Marvian and Lidar, "there is no advantage to using such codes." Here, we answer positively the specific question as to whether a Hamiltonian consisting of non-commuting two-local terms can be useful in combating single-qubit errors. While our claims go somewhat beyond single-qubit errors, we are not making broader claims about fault tolerance or quantum memories.

One potential way to get around the no-go result is to try subsystem codes. We concentrate on stabilizer subsystem codes [2,3], which encode information only in a subset of the qubits used by related stabilizer codes. Subsystem codes offer great flexibility in designing mechanisms to support robust quantum computation, because the effect of errors on the unused logical qubits is not a concern [11, 12]. Subsystem codes support routines that make use of the subsystem structure to break stabilizer terms into lower-weight terms. However, unlike the commuting case, arising from stabilizer codes, in which the Hamiltonians are automatically gapped [9], there is no known general theorem on gaps for non-commuting Hamiltonians.

Nevertheless, we show that indeed one can obtain single-qubit error suppression by 
encoding in the ground subspace of a Hamiltonian consisting of a weighted sum of noncommuting two-local terms from the gauge generators of a Bacon-Shor code [2] or one of Bravyi's generalized-Bacon-Shor codes [3]. Both of the Bacon-Shor and the generalizedBacon-Shor codes satisfy the Knill-Laflamme condition exactly, and therefore there is no "induced degeneracy splitting" that leads to the difficulties identified by Marvian and Lidar in the commuting case [1]. In particular, these codes do not come up against their generalization of the no-hiding theorem.

We explore three specific examples in detail, the $[[4,1,2]]$ Bacon-Shor code and the $[[6,2,2]]$ and $[[16,2,3]]$ generalized-Bacon-Shor codes. For each of these codes, we compute the energy separation between the ground subspace and the orthogonal subspaces. (We refer to the "energy separation" between the ground subspace and the orthogonal subspaces as opposed to the "gap" so as to avoid confusion with the "gap" in an adiabatic computation, especially when discussing error suppression in the adiabatic context.) We provide a general technique to reduce the dimension of the Hilbert space that needs to be considered, which eases the calculation of the energy separation for arbitrary stabilizer subsystem codes.

The first code we consider provides the simplest example in which the construction works. The second gives a more compact encoding and enables a reduction in the number of higher-order terms in encodings of commonly used adiabatic Hamiltonians such as the Ising Hamiltonians common in adiabatic quantum optimization and quantum annealing. These codes are part of a family of codes that provide more and more compact encodings, and greater reductions in the number of higher-order terms, but for which the energy separation becomes increasingly computationally expensive to compute. The third code provides an example that suppresses 2-qubit errors and provides a good example in which to see the workings of the dimension reduction algorithm. For the first two codes, we perform a numerical analysis of the open-system dynamics with the spin-boson error model, confirming exponential suppression of single-qubit errors. We also remark briefly on the robustness of this approach to control errors.

We expect this approach to find application in improved robustness of near-term implementations of quantum storage, quantum annealing, and adiabatic quantum computing, and perhaps in quantum networks $[13,14]$.

\section{BRIEF REVIEW OF QUANTUM CODES AND ERROR SUPPRESSION}

The general strategy for combating local errors is to spread the "logical" or "computational" information across physical qubits. A particularly common way of doing so is through block codes. A quantum [[n,k]]-block code is a $2^{k}$-dimensional subspace of a $2^{n}$ dimensional Hilbert space, which enables $k$ logical qubits to be encoded in $n$ physical qubits. In this review section, we first give a brief review of the most common type of quantum error-correcting codes, stabilizer codes, and then discuss how such codes are used to achieve error suppression. We then describe subsystem codes, concentrating on 
stabilizer subsystem codes, codes that can be viewed as stabilizer codes in which only some of the logical qubits are used to encode quantum information.

\section{A. Stabilizer codes}

Consider the generalized Pauli group $\mathcal{P}_{G}$ acting on an $n$ qubit system. Let $S$ be a subgroup of $\mathcal{P}_{G}$ generated by $r$ independent, commuting generators. The subgroup $S$ defines a code space $C$, the stabilizer subspace of $S$, the joint +1 -eigensubspace of all elements in $S$. The dimension of $C$ is $2^{k}$ where $k=n-r$.

Let $C_{\mathcal{P}_{G}}(S)$ be the centralizer of $S$, the set of elements in $\mathcal{P}_{G}$ that commute with all elements of $S$. We define $k$ logical qubits encoded in $C$ by specifying the logical Pauli operators in $\mathcal{P}_{G}$ that define the qubits. Any choice of elements outside $S$, but commuting with $S$, that satisfy the Pauli commutation relations works. There are a number of different ways of encoding, each with its own choice of logical operators.

The distance $d$ of a quantum error-correcting code is the minimum number of singlequbit error by which an element of the code space can be transformed into an orthogonal element of the code space. The weight of a Pauli error $e \in \mathcal{P}_{G}$ is the number of qubits on which a non-identity Pauli transformation acts. An $[[n, k, d]]$-quantum code can detect up to weight $d-1$ errors, and can correct errors of weight up to $t$ satisfying $2 t<d$.

We now give the $[[4,2,2]]$-stabilizer code as an example since it will set us up well for discussing the $[[4,1,2]]$-subsystem code. Consider a 4-qubit system consisting of qubits $q_{1,1}, q_{1,2}, q_{2,1}$, and $q_{2,2}$. Let $C$ be the joint +1 -eigensubspace of the two stabilizer generators

$$
\begin{aligned}
& S^{X}=X_{1,1} X_{1,2} X_{2,1} X_{2,2} \\
& S^{Z}=Z_{1,1} Z_{2,1} Z_{1,2} Z_{2,2} .
\end{aligned}
$$

We may define logical operators that define two encoded qubits as:

$$
\begin{array}{ll}
\tilde{X}_{L 1}=X_{1,1} X_{2,1}, & \tilde{Z}_{L 1}=Z_{1,1} Z_{1,2} \\
\tilde{X}_{L 2}=X_{1,1} X_{1,2}, & \tilde{Z}_{L 2}=Z_{1,1} Z_{2,1} .
\end{array}
$$

The reader may check that $X_{L 1}, Z_{L 1}, X_{L 2}$, and $Z_{L 2}$ are in $C_{\mathcal{P}_{G}}(S)$, satisfy the Pauli commutation relations, and that the code distance is 2 . A useful property of stabilizer codes is that any operator that is the product of a logical operator and stabilizers behaves on the code space in exactly the same way as the logical operator behaves on the code space since the stabilizers commute with logical operators.

\section{B. Error suppression with stabilizer codes}

Let $H_{0}(t)$ be a problem Hamiltonian acting on an $n$-qubit system. Given an $[[n, k, d]]$ stabilizer code, the Hamiltonian

$$
H(t)=H_{L}(t)+E_{P} H_{\text {supp }}
$$


implements error suppression, where $E_{P}$ is the penalty weight, $H_{\text {supp }}$ is the negative of the sum of $M$ stabilizers, and $H_{L}(t)$ is obtained from $H_{0}(t)$ by replacing all operators with their logical counterpart. Any error of weight less than $d$ will take the state out of the +1 -eigensubspace of at least one stabilizer, and thus receives a penalty from $H_{\text {supp }}$. As an example, the problem Hamiltonian

$$
H_{0}(t)=a(t) \sum_{i=1}^{n} X_{i}+b(t)\left(\sum_{i=1}^{n} h_{i} Z_{i}+J_{i j} Z_{i} Z_{j}\right)
$$

becomes the encoded Hamiltonian

$$
H(t)=a(t) \sum_{i=1}^{n} \tilde{X}_{i}+b(t)\left(\sum_{i=1}^{n} h_{i} \tilde{Z}_{i}+J_{i j} \tilde{Z}_{i} \tilde{Z}_{j}\right)+E_{P} H_{\text {supp }}
$$

By construction, the logical Hamiltonian $H_{L}(t)$ commutes with the error suppression

term $H_{\text {supp }}$, so in the error-free case, the logical dynamics of the code space under $H(t)$ is that of the original space under $H(t)$.

The Hamiltonian $H_{\text {supp }}$ is 4-local for the $[[4,1,2]]$ code, since its stabilizers act on all four physical qubits. The energy separation of $H_{\text {supp }}$ is $E_{P}$, because the sum of the two commuting stabilizers has eigenvalues $-1,0,1$. In the next subsection, we will see how the 4-local stabilizers can be broken into 2-local gauge operators by encoding the quantum information in a subsystem instead of a subspace.

\section{Subsystem codes}

Subsystem codes decompose the Hilbert space into $\mathcal{H}=\mathcal{H}_{L} \otimes \mathcal{H}_{G}$, where $\mathcal{H}_{L}$ is the subsystem of logical qubits that store information, and $\mathcal{H}_{G}$ is the subsystem of the gauge qubits. We concentrate on stabilizer subsystem codes [2, 15], a generalization of stabilizer codes, particularly 2D generalized-Bacon-Shor codes. Stabilizer subsystem codes can be viewed as stabilizer codes in which only some of the logical qubits are used to encode quantum information.

A 2D generalized-Bacon-Shor code is specified by a 2D array, or matrix, $M$ with entries in $\mathbb{Z}_{2}$. For each 1 in the matrix, we have a physical qubit. The matrix defines a $[[n, k, d]]$ code where $n$ is the number of nonzero matrix elements in $M, k$ is the rank of $M$, and $d$ is the minimum distance of the two classical codes defined by the rows and columns of $M$, respectively.

The gauge group $\mathcal{G}$ is generated by $X X$ operators for every pair of qubits in the rows of $M$ and $Z Z$ operators for every pair of qubits in the columns of $M$. There is redundancy in this generating set. A commonly used non-redundant generating set is the set of generators corresponding to nearest neighbor pairs. The stabilizer subgroup of the code is the center of $\mathcal{G}$, the set of elements of $\mathcal{G}$ that commute with all elements of $\mathcal{G}$. The reader can check that the stabilizer subgroup consists of all products of 


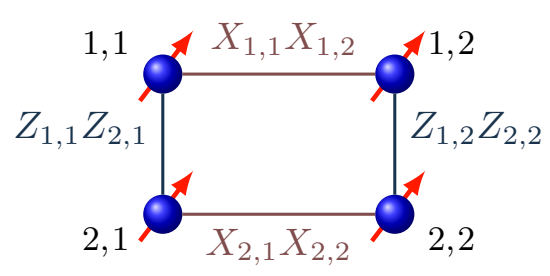

FIG. 1. The gauge generators of the Bacon-Shor [[4,1,2]]-code consist of two-qubit operators of type $X X(Z Z)$ that couple qubits in a row (column).

$X$ operators corresponding to linearly dependent sets of rows and all products of $Z$ operators corresponding to linearly dependent sets of columns.

The logical operators are chosen to be elements of $\mathcal{C}(\mathcal{G}) \backslash \mathcal{G}$, elements of the centralizer of the gauge group that are not in the gauge group. Just as in the stabilizer code case, there is freedom in the choice of logical operators. We define $k$ logical qubits by specifying logical Pauli operators in $\mathcal{C}(\mathcal{G}) \backslash \mathcal{G}$ that satisfy the Pauli commutation relations.

It is useful to define auxiliary qubits, subspaces of the gauge subspace that are not in the stabilizer subspace. These qubits will not be used to encode computational information, but will be useful in analyzing subsystem codes.

Example: The [[4, 1,2]]-stabilizer subsystem code.

The most familiar subsystem code is the Bacon-Shor $[[9,1,3]]$-code, the smallest Bacon-Shor code that corrects single-qubit errors. For error suppression, codes that only detect errors can be used, allowing us to consider the smallest error-detecting Bacon-Shor code, the [[4, 1,2]]-code, which detects single-qubit errors. This code has been used before, by Brell et al. [16], as a gadget to obtain the Hamiltonians for the toric code and Kitaev's quantum double models as the low-energy limits of two-body Hamiltonians.

The gauge group for the $[[4,1,2]]$-code is

$$
\mathcal{G}=\left\langle G_{1}^{X}, G_{2}^{X}, G_{1}^{Z}, G_{2}^{Z}\right\rangle
$$

which is generated by the gauge generators

$$
\begin{aligned}
& G_{1}^{X}=X_{1,1} X_{1,2}, \quad G_{2}^{X}=X_{2,1} X_{2,2}, \\
& G_{1}^{Z}=Z_{1,1} Z_{2,1}, \quad G_{2}^{Z}=Z_{1,2} Z_{2,2},
\end{aligned}
$$

shown in Figure 1. The stabilizer subgroup is generated by

$$
\begin{aligned}
& S^{X}=X_{1,1} X_{1,2} X_{2,1} X_{2,2} \\
& S^{Z}=Z_{1,1} Z_{2,1} Z_{1,2} Z_{2,2} .
\end{aligned}
$$

The reader will recognize these stabilizers as those defining the $[[4,2,2]]$-stabilizer code. The $[[4,1,2]]$-subsystem code encodes information in only one of the logical qubits of 
the $[[4,2,2]]$-stabilizer code. One choice of logical operators is

$$
X_{L}=X_{1,1} X_{2,1}, \quad Z_{L}=Z_{1,1} Z_{1,2} .
$$

A convenient choice of auxiliary operators is

$$
X_{A}=G_{1}^{X}=X_{1,1} X_{1,2}, \quad Z_{A}=G_{1}^{Z}=Z_{1,1} Z_{2,1} .
$$

These operators would have defined a logical qubit in a stabilizer code, but in the subsystem code this qubit is not used to encode computational information.

Examples of larger subsystem codes with more complex structure will be given in later sections.

\section{TWO-LOCAL HAMILTONIANS FOR ERROR SUPPRESSION}

Given a Hamiltonian $H(t)$ we wish to encode to suppress errors and a specific subsystem code, we create an encoded Hamiltonian $H_{E}(t)=H_{L}(t)+H_{\text {supp }}$, where $H_{L}(t)$ is obtained from $H$ by replacing each operator in $H$ with the corresponding operator for the code and where $H_{\text {supp }}$ is a weighted sum of the gauge operators. Because the logical operators commute with the gauge group, the evolution of the encoded subspace under $H_{E}(t)$ is that of $H(t)$ acting on an unencoded system, so the dynamics are correct.

Unlike the stabilizer code case, in which all the terms of $H_{\text {supp }}$ commute and therefore the Hamiltonian is known to be gapped, we must check that $H_{\text {supp }}$ has an energy separation between the ground subspace and orthogonal subspaces. Because the gauge group of any stabilizer subsystem code is generated by two-local operators, the suppression term $H_{\text {supp }}$ is always two local. As we will see, whether $H_{L}$ is two-local depends on properties of $H$ and of the subsystem code.

\section{A. Suppression with the Bacon-Shor $[[4,1,2]]$-code}

Consider an error-suppressing Hamiltonian that is a weighted sum of the gauge generators, where the weights $\lambda_{i}$ and $\eta_{i}$ are real:

$$
\begin{aligned}
H_{\text {supp }} & =-\lambda_{1} G_{1}^{X}-\eta_{1} G_{1}^{Z}-\lambda_{2} G_{2}^{X}-\eta_{2} G_{2}^{Z} \\
& =-\lambda_{1} X_{A}-\eta_{1} Z_{A}-\lambda_{2} S^{X} X_{A}-\eta_{2} S^{Z} Z_{A} \\
& =-\left(\lambda_{1}+\lambda_{2} S^{X}\right) X_{A}-\left(\eta_{1}+\eta_{2} S^{Z}\right) Z_{A},
\end{aligned}
$$

where the last line uses the relation $G_{2}^{X}=S^{X} X_{A}$ and $G_{2}^{Z}=S^{Z} Z_{A}$. This Hamiltonian, being a linear combination of the gauge generators, commutes with the stabilizers and logical operators. It takes a block diagonal form in the eigenbasis of the stabilizers. The 


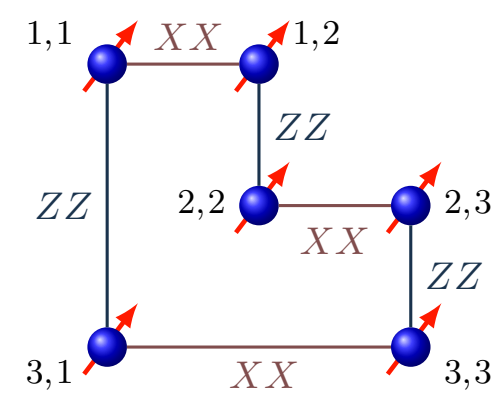

FIG. 2. The gauge generators of Bravyi's [[6, 2,2]]-code formed using 6 physical qubits placed within a $3 \times 3$ lattice. The gauge generators consist two-qubit operators of type $X X(Z Z)$ coupling qubits in a row (column).

energy separation of $H_{\text {supp }}$ is a function of $\lambda_{i}$ and $\eta_{i}$, which can be calculated exactly in this case. For fixed values of the stabilizers, we have

$$
H_{\text {supp }}^{(x, z)}=-\left(\lambda_{1}+\lambda_{2} x\right) X_{A}-\left(\eta_{1}+\eta_{2} z\right) Z_{A}
$$

where $x, z= \pm 1$ are the eigenvalues of $S^{X}$ and $S^{Z}$, respectively. Any detectable error inevitably changes the value of the stabilizers, and is suppressed by the energy separation of $H_{\text {supp }}$. The eigenvalues of this Hamiltonian are

$$
E_{\mathrm{supp}}^{(x, z)}= \pm \sqrt{\left(\lambda_{1}+x \lambda_{2}\right)^{2}+\left(\eta_{1}+z \eta_{2}\right)^{2}}
$$

For the case where $\lambda_{1}, \lambda_{2}>0$ and $\eta_{1}, \eta_{2}>0$, the ground subspace of $H_{\text {supp }}^{\left(x^{\star}, z^{\star}\right)}$ with $x^{\star}=$ $z^{\star}=1$ has energy strictly smaller than that of $E_{\text {supp }}^{(x, z)}$ with $(x, z) \neq(1,1)$. Any singlequbit error on logical information encoded in the ground state of $H_{\text {supp }}^{\left(x^{\star}, z^{\star}\right)}$ is suppressed, because it raises the energy by changing the value of $x^{\star}$ or $z^{\star}$. For $\lambda_{1}=\lambda_{2}=\eta_{1}=\eta_{2}=$ 1 , the eigenvalues are $\pm \sqrt{8}, \pm 2,0$, so the gap is $2(\sqrt{2}-1)$. Thus, unlike in the stabilizer code case, a two-local Hamiltonian can suppress arbitrary single-qubit errors.

\section{B. Suppression with the Bacon-Shor $[[6,2,2]]$-code}

We now turn to Bravyi's [[6, 2,2]] generalized-Bacon-Shor code [3]. It has the advantage of being more compact, encoding two logical qubits in six physical qubits, and has the further advantage, as we will see, that certain two-qubit logical operators can be implemented using operations involving only two physical qubits.

The following matrix defines Bravyi's $[[6,2,2]]$-code

$$
M=\left(\begin{array}{lll}
1 & 1 & 0 \\
0 & 1 & 1 \\
1 & 0 & 1
\end{array}\right)
$$


The gauge group of Bravyi's code is

$$
\mathcal{G}=\left\langle G_{1}^{X}, G_{2}^{X}, G_{3}^{X}, G_{1}^{Z}, G_{2}^{Z}, G_{3}^{Z}\right\rangle
$$

generated by the gauge generators as shown in Figure 2:

$$
\begin{array}{ccc}
G_{1}^{X}=X_{1,1} X_{1,2}, & G_{2}^{X}=X_{2,2} X_{2,3}, & G_{3}^{X}=X_{3,1} X_{3,3} \\
G_{1}^{Z}=Z_{1,1} Z_{3,1}, & G_{2}^{Z}=Z_{1,2} Z_{2,2}, & G_{3}^{Z}=Z_{2,3} Z_{3,3} .
\end{array}
$$

The center of $\mathcal{G}$ is generated by the stabilizers

$$
S^{X}=G_{1}^{X} G_{2}^{X} G_{3}^{X}, \quad S^{Z}=G_{1}^{Z} G_{2}^{Z} G_{3}^{Z} .
$$

One choice for the logical operators is

$$
\begin{array}{ll}
X_{L 1}=X_{2,3} X_{3,3}, & Z_{L 1}=Z_{3,1} Z_{3,3}, \\
X_{L 2}=X_{1,2} X_{2,2}, & Z_{L 2}=Z_{1,1} Z_{1,2} .
\end{array}
$$

The auxiliary logical operators can be chosen to be the gauge elements

$$
\begin{array}{ll}
X_{A 1}=X_{3,1} X_{3,3}=G_{3}^{X}, & Z_{A 1}=Z_{2,3} Z_{3,3}=G_{3}^{Z}, \\
X_{A 2}=X_{1,1} X_{1,2}=G_{1}^{X}, & Z_{A 2}=Z_{1,2} Z_{2,2}=G_{2}^{Z} .
\end{array}
$$

The original gauge generators can be expressed using the stabilizers and the unused logical operators

$$
\begin{gathered}
G_{1}^{X}=X_{A 2}, \quad G_{2}^{X}=S^{X} X_{A 1} X_{A 2}, \quad G_{3}^{X}=X_{A 1}, \\
G_{1}^{Z}=S^{Z} Z_{A 1} Z_{A 2}, \quad G_{2}^{Z}=Z_{A 2}, \quad G_{3}^{Z}=Z_{A 1} .
\end{gathered}
$$

Consider an error-suppressing Hamiltonian that is a weighted sum of the gauge generators

$$
H_{\text {supp }}=-\lambda_{1} G_{3}^{X}-\lambda_{2} G_{1}^{X}-\lambda_{1}^{\prime} G_{3}^{Z}-\lambda_{2}^{\prime} G_{2}^{Z}-\eta G_{2}^{X}-\eta^{\prime} G_{1}^{Z},
$$

where all coefficients are positive real numbers. For fixed values of the stabilizers $S^{X}=x$ and $S^{Z}=z$, the Hamiltonian (18) can be written in terms of the auxiliary logical operators

$$
H_{\text {supp }}^{(x, z)}=-\lambda_{1} X_{A 1}-\lambda_{2} X_{A 2}-\lambda_{1}^{\prime} Z_{A 1}-\lambda_{2}^{\prime} Z_{A 2}-\eta x X_{A 1} X_{A 2}-\eta^{\prime} z Z_{A 1} Z_{A 2} .
$$

We now demonstrate that, as for the $[[4,1,2]]$ code, the energy separation of the above Hamiltonian for the $[[6,2,2]]$ code is nonzero for typical choices of the weights. To suppress errors detectable by the code, the ground states of $H_{\text {supp }}$ should also be eigenstates of the stabilizers with fixed eigenvalues $x=x^{\star}$ and $z=z^{\star}$. Consequently, the Hamiltonian $H_{\text {supp }}^{(x, z)}$ must depend on the values of $x$ or $z$, so we must have $\eta \neq 0$ and 
$\eta^{\prime} \neq 0$. In general, to achieve error suppression, none of the coefficients in Eq. (19) can be zero. For example, we must have $\lambda_{1} \neq 0$; otherwise, the eigenvalues of $H_{\text {supp }}^{(x, z)}$ would not depend on the value of $x$. We can show this by simply flipping the sign of the term with coefficient $-\eta x$ by applying the unitary transformation $Z_{A 1} H_{\text {supp }}^{(x, z)} Z_{A 1}^{\dagger}$, without changing anything else.

The general case can be solved directly. A particular choice of the error-suppressing Hamiltonian that can be solved easily is

$$
H_{\text {supp }}^{(x, z)}=-\lambda\left(X_{A 1}+X_{A 2}+Z_{A 1}+Z_{A 2}\right)-\eta\left(x X_{A 1} X_{A 2}+z Z_{A 1} Z_{A 2}\right),
$$

where $\lambda, \eta>0$. In the Bell basis of the two auxiliary logical qubits, we have

$$
H_{\text {supp }}^{\left(s_{ \pm}\right)}=-2\left(\begin{array}{cccc}
\eta s_{+} & \lambda & \lambda & 0 \\
\lambda & \eta s_{-} & 0 & 0 \\
\lambda & 0 & -\eta s_{-} & 0 \\
0 & 0 & 0 & -\eta s_{+}
\end{array}\right),
$$

where $s_{ \pm}=(x \pm z) / 2 \in\{-1,0,1\}$, and the singlet state is always decoupled from the other states. The eigenvalues of this matrix are $\left\{-\eta s_{+} \pm \sqrt{8 \lambda^{2}+\eta^{2}}, 2 \eta s_{+}, 0\right\}$, for $s_{+}= \pm 1$ and $s_{-}=0$, and $\left\{ \pm 2 \sqrt{2 \lambda^{2}+\eta^{2}}, 0,0\right\}$, for $s_{+}=0$ and $s_{-}= \pm 1$. Thus, the ground subspace for the case $x=z=1$ has the lowest energy $-\left(\eta+\sqrt{8 \lambda^{2}+\eta^{2}}\right)$, and the two ground states for $x=-z= \pm 1$ have the second-lowest energy $-2 \sqrt{2 \lambda^{2}+\eta^{2}}$. The gap is $\eta+\sqrt{8 \lambda^{2}+\eta^{2}}-2 \sqrt{2 \lambda^{2}+\eta^{2}}$. The gap goes to $\eta$ for $\lambda \rightarrow \infty$, and is zero for $\lambda \rightarrow 0$. It equals to $4-2 \sqrt{3} \simeq 0.536$ for $\lambda=\eta=1$.

\section{Reducing number of higher-order terms in logical Hamiltonians}

While the error suppression term coming from these subsystem code constructions is two local, the logical Hamiltonian may contain higher-order terms. For example, an Ising Hamiltonian has $Z Z$ terms. The corresponding logical Ising Hamiltonian, implemented using two two-local logical $Z$ operators, would in general be four local.

In addition to compactness, another advantage of the $[[6,2,2]]$-code over the $[[4,1,2]]$ code is that the logical operators acting on two qubits encoded together are two-local. Because the stabilizer terms commute with the encoded logical Hamiltonian, $X_{L 1} X_{L 2}$ can be implemented using $X_{1,1} X_{3,1}$ and $Z_{L 1} Z_{L 2}$ can be implemented using $Z_{2,2} Z_{2,3}$ since

$$
X_{L 1} X_{L 2}=S^{X} X_{1,1} X_{3,1}, \quad Z_{L 1} Z_{L 2}=S^{Z} Z_{2,2} Z_{2,3},
$$

so certain two-qubit logical operators, those acting on two logical qubits encoded together, can be implemented with two-local interactions only. When multiple qubits are encoded using multiple copies of the code, two-qubit logical operators acting on logical qubits encoded by different copies will still need four-body interactions. Nevertheless, this code has an advantage over the $[[4,1,2]]$-code in which every logical qubit 
is encoded separately so one needs four-body interactions to implement every two-qubit logical operation.

These codes are the first two examples of codes in a family of $[[2 k+2, k, 2]]$-codes specified by $(k+1) \times(k+1)$-matrices $M$ with row $i$ containing $1 \mathrm{~s}$ only in positions $i$ and $i+1$ (where the $k+1$ st row contains $1 \mathrm{~s}$ in positions $k+1$ and 1 ). Unfortunately, the energy separation becomes more and more computationally intensive to compute as $k$ increases.

\section{Generalized construction}

We have seen that it is useful to write the error-suppressing Hamiltonian in terms of auxiliary operators and stabilizers. Doing so removes the degeneracy in $H_{\text {supp }}$ and reduces the size of the Hilbert space under consideration, enabling numerical calculation of the energy separation between the code subspace and orthogonal subspaces. Such a reduction is always possible for generalized-Bacon-Shor codes; in Appendix A we give an algorithm that provides a systematic way of finding auxiliary operators satisfying the standard commutation relations that, together with the stabilizer operators, generate the gauge group.

We made use of this algorithm to compute the energy separation for a $[[16,2,3]]$ generalized-Bacon-Shor code (see Appendix A for more details). Again, we may encode information in the ground subspace with all stabilizers taking value +1 . Then, for $\lambda=1$, when the error suppression term is precisely the (unweighted) sum of all gauge generators, the ground subspace energy is -13.83 and the energy separation between the code space and the orthogonal subspaces is 0.33 .

We can apply this construction to generalized-Bacon-Shor codes that encode a larger number of qubits at once, thus increasing the proportion of logical operators that can be implemented using only two-local interactions. However, for these larger codes, computing the energy separation becomes computationally prohibitive. As was shown in [17] using exact diagonalizations, Greens function Monte Carlo simulations, and high-order perturbation theory, the energy separation of a Bacon-Shor code (or the quantum compass model) vanishes exponentially in the size of the code. An open question is whether it is possible to obtain a nonzero asymptotic separation for some family of generalized-Bacon-Shor codes.

\section{E. Robustness to implementation errors}

Our analysis shows that effective error suppression can be obtained using a variety of different weighted sums of the gauge generators as the $H_{\text {supp }}$ term. For this reason, this approach is highly robust to control errors that result in imprecise implementation of the weights of these terms. Furthermore, when the weight of any implementation error in the logical Hamiltonian is less than the code distance, these errors are suppressed 
by $H_{\text {supp. }}$. This robustness does not yield fault tolerance, but does mean that this error suppression approach can be useful in near-term implementations of quantum computational devices and quantum storage.

\section{NUMERICAL ANALYSIS OF EFFECTIVENESS OF ERROR SUPPRESSION WITH SPIN-BOSON NOISE MODEL}

We perform a numerical analysis of the effectiveness these codes in suppressing errors due to qubits coupling to individual baths. Specifically, we show that decoherence effects on qubits coupled to bosonic baths with Ohmic spectra are exponentially suppressed when the energy separation of $H_{\text {supp }}$ becomes larger than $k_{\mathrm{B}} T$. A physical interpretation for that condition is that the bath modes, which resonate with the transition frequencies of the system to higher-energy states, are close to the vacuum state at the temperature $T$ and thus are not capable of driving such transitions.

\section{A. Spin-boson noise model}

The spin-boson error model [18] we use generalizes the one used in [19] in which individual system qubits are coupled to independent baths, but via all Pauli operators not just $Z$ operators,

$$
H=H_{\text {supp }}+H_{B}+\sum_{k=1}^{n}\left(X_{k} \otimes B_{k}^{X}+Y_{k} \otimes B_{k}^{Y}+Z_{k} \otimes B_{k}^{Z}\right),
$$

where $H_{B}$ is the bath Hamiltonian, $B_{k}^{X}$ is the bath operator coupled to the $X$ operator of the $k$ th qubit, and similarly for $B_{k}^{Y}$ and $B_{k}^{Z}$ (see the Appendix B for more details).

We assume that the bath spectral functions $C_{\mathrm{bath}}(\omega)$ are the same for all physical qubits. Its Fourier transformation takes the following form for the Ohmic case,

$$
\widetilde{C}_{\text {bath }}(\omega)=\frac{2 \pi \hbar^{2} \chi \omega e^{-|\omega| / \omega_{c}}}{1-e^{-\hbar \omega / k_{\mathrm{B}} T}}
$$

where $T$ is the temperature, $\omega_{c}$ is the cutoff frequency, and $\chi$ is a dimensionless parameter proportional to the product of the system-bath coupling strength and the bath spectral density. For $\omega<0$ (a transition that raises the energy of the system) and $|\omega|$ sufficiently large, the term in the denominator becomes most salient, yielding exponential suppression of decoherence. In our analysis, we use the parameters from [19]: $\chi=3.18 \times 10^{-4}, \omega_{c}=8 \pi \times 10^{9} \mathrm{rad} / \mathrm{s}$, and $k_{\mathrm{B}} T / \hbar=2.2 \times 10^{9} \mathrm{rad} / \mathrm{s}($ at $17 \mathrm{mK})$.

\section{B. Numerical analysis of error suppression}

After going to the interaction picture of $H_{\text {supp }}$, we simulate the open-system dynamics of the qubits using the Markov approximation. The bath correlation function $\widetilde{C}_{\text {bath }}(\omega)$ 

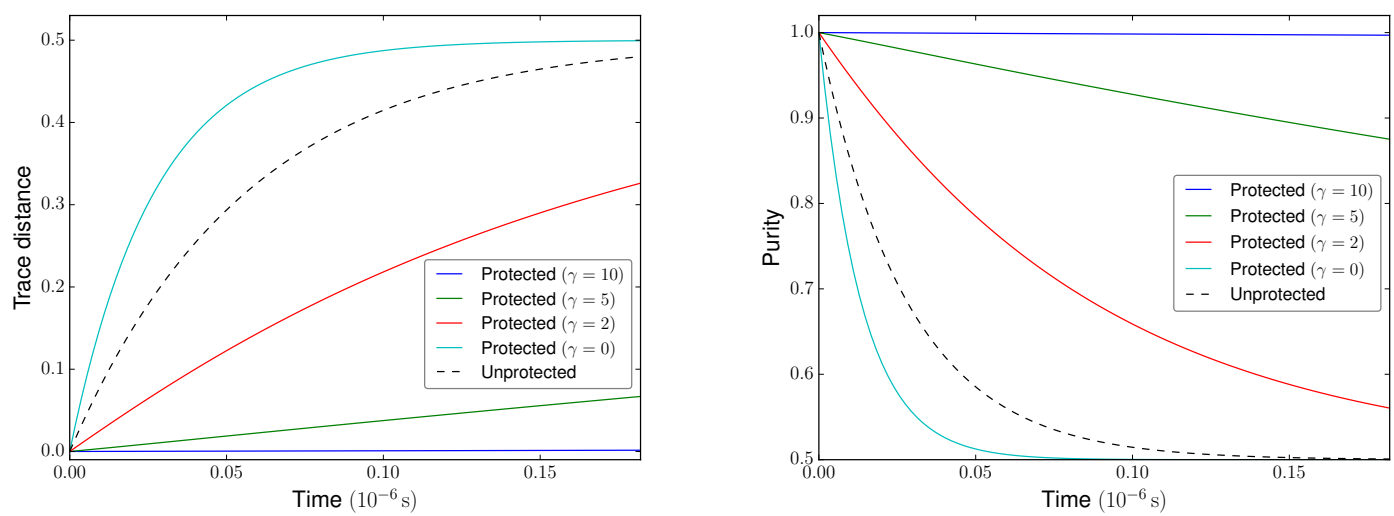

FIG. 3. Simulations of the open-system dynamics of a single logical qubit, encoded in the $[[4,1,2]]$ subsystem code, under single-qubit noise for different penalty weights $\gamma=\lambda / k_{\mathrm{B}} T$. The initial state of the logical qubit is set to be the state $|+\rangle_{L}$, the +1 eigenstate of $X_{L}$. (Left) Trace distance between the initial and evolved state. (Right) Purity of the evolved state.

determines the transition rate between two states in two different energy eigenspaces of $H_{\text {supp }}$. A transition is suppressed when the energy increase is significantly greater than $k_{\mathrm{B}} T / \hbar$.

For various codes, we consider an error-suppressing Hamiltonian proportional to the sum over a generating set $\mathcal{R}$ for $\mathcal{G}, H_{\text {supp }}=-\lambda \sum_{G \in \mathcal{R}} G$. In Figure 3 we show results for the $[[4,1,2]]$ subsystem codes at various implementable values of $\gamma=\lambda / k_{\mathrm{B}} T$. Error suppression is helpful once $\gamma$ is larger than a threshold value of $\simeq 0.6$. Below that value, the system is more vulnerable to decoherence because the encoded logical state is an entangled state involving more physical qubits than the unprotected state, and thus is exposed to more noise.

Figure 4 shows, under the same noise model for various values of $\gamma$, the purity of the state and the trace distance between the evolved and ideal states for two logical qubits, initially in a Bell state $(|00\rangle+|11\rangle) / \sqrt{2}$, encoded together in a single block of the $[[6,2,2]]$ code.

We also examined how well the two codes preserve entanglement. Figure 5 shows the entanglement of formation of two logical qubits, initially in a Bell state, each encoded separately using (Left) the $[[4,1,2]]$ code and (Right) the $[[6,2,2]]$ code. Entanglement is better preserved by the $[[4,1,2]]$ code due to the larger energy separation between its ground subspace and orthogonal subspaces.

\section{CONCLUSION}

We have shown the effectiveness of a two-local Hamiltonian based on subsystem codes in suppressing single-qubit errors, thereby avoiding the no-go theorem that exists 

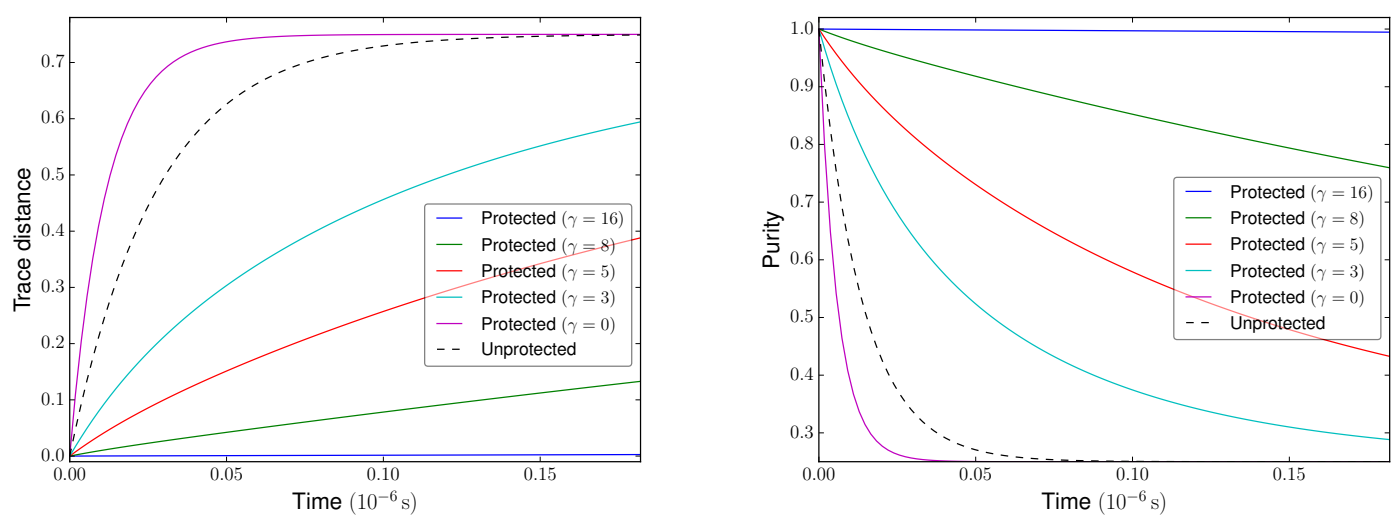

FIG. 4. Simulations of the open-system dynamics of the two logical qubits, encoded in the $[[6,2,2]]$ subsystem code, under single-qubit noise for different penalty weights $\gamma=\lambda / k_{\mathrm{B}} T$. The initial state of the logical qubits is the Bell state $(|00\rangle+|11\rangle) / \sqrt{2}$. (Left) Trace distance between the initial and evolved state. (Right) Purity of the evolved state.
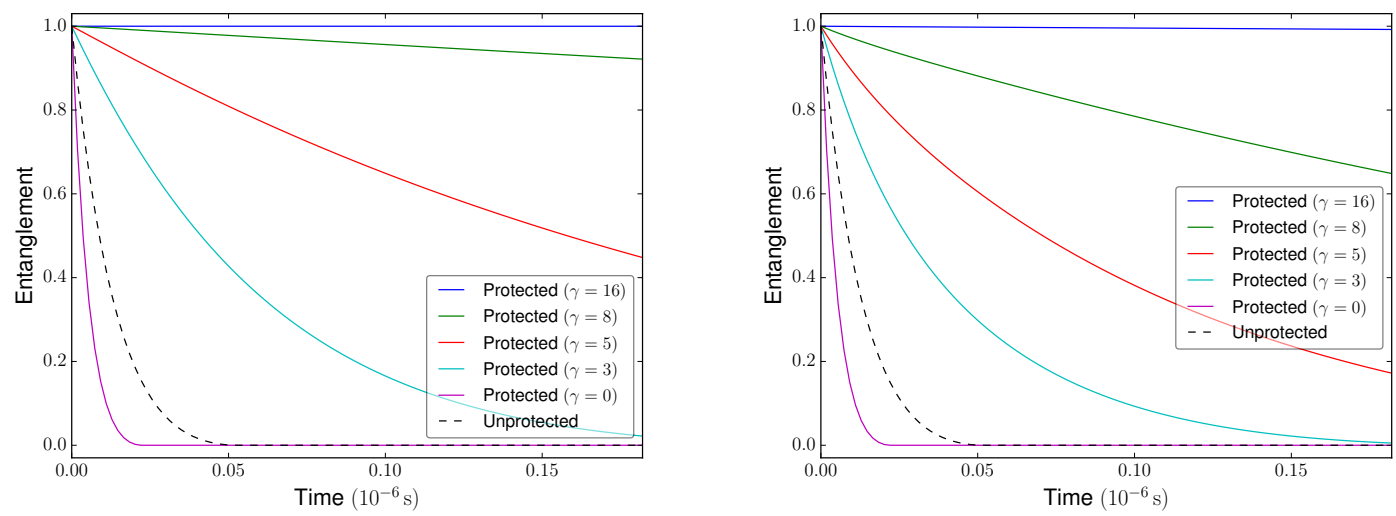

FIG. 5. Simulations of the time evolution of entanglement between two logical qubits. The initial state is set to be the Bell state $(|00\rangle+|11\rangle) / \sqrt{2}$ of the logical qubits. (Left) Two qubits encoded separately using the $[[4,1,2]]$ code. (Right) Two qubits encoded together using the $[[6,2,2]]$ code.

in the stabilizer case. We analyzed the energy separation between the ground subspace and orthogonal subspaces for some simple codes. As part of this analysis, we developed a general technique to reduce the dimension of the Hilbert space that needs to be considered, which eases the calculation of the energy separation for arbitrary stabilizer subsystem codes. We discussed the robustness of the subsystem-code-based error suppression schemes with respect to implementation errors. We also numerically evaluated the effectiveness of these schemes in suppressing errors coming from individual qubit 
interactions with a spin-boson bath. One advantage of generalized-Bacon-Shor codes is the ability to implement certain two-qubit logical operators by involving only two physical qubits.

We expect the subsystem error suppression approach discussed here to find applications in providing greater robustness for near-term quantum annealers, and also in the storage of quantum information, for example in quantum networks. More sophisticated techniques that would enable deeper analysis or more efficient numerical investigation of subsystem code error suppression would be welcome. Of particular interest would be techniques that enabled a scaling analysis of the energy separation for families of subsystem codes, with the hopes of finding families of subsystem codes with asymptotically advantageous scaling of the energy separation or ruling out such a possibility.

\section{ACKNOWLEDGMENTS}

The authors would like to acknowledge support from the NASA Advanced Exploration Systems program and NASA Ames Research Center. This work was supported in part by the AFRL Information Directorate under Grant F4HBKC4162G001, the Office of the Director of National Intelligence (ODNI), and the Intelligence Advanced Research Projects Activity (IARPA), via IAA 145483. The views and conclusions contained herein are those of the authors and should not be interpreted as necessarily representing the official policies or endorsements, either expressed or implied, of ODNI, IARPA, AFRL, or the US Government. The US Government is authorized to reproduce and distribute reprints for Governmental purpose notwithstanding any copyright annotation thereon.

[1] Iman Marvian and Daniel A. Lidar, "Quantum error suppression with commuting Hamiltonians: Two local is too local," Physical Review Letters 113, 260504 (2014).

[2] Dave Bacon, "Operator quantum error-correcting subsystems for self-correcting quantum memories," Physical Review A 73, 012340 (2006).

[3] Sergey Bravyi, "Subsystem codes with spatially local generators," Physical Review A 83, 012320 (2011).

[4] Michael Nielsen and Isaac Chuang, Quantum Computing and Quantum Information (Cambridge University Press, Cambridge, 2001).

[5] E. G. Rieffel and W. Polak, Quantum Computing: A Gentle Introduction (MIT Press, Cambridge, MA, 2011).

[6] Barbara M. Terhal, "Quantum error correction for quantum memories," Reviews of Modern Physics 87, 307-346 (2015).

[7] Stephen P. Jordan, Edward Farhi, and Peter W. Shor, "Error-correcting codes for adiabatic quantum computation," Physical Review A 74, 052322 (2006). 
[8] Kevin C. Young, Mohan Sarovar, and Robin Blume-Kohout, "Error suppression and error correction in adiabatic quantum computation: Techniques and challenges," Physical Review X 3, 041013 (2013).

[9] Sergey Bravyi and Mikhail Vyalyi, "Commutative version of the local Hamiltonian problem and common eigenspace problem," Quantum Info. Comput. 5, 187-215 (2005).

[10] D. Aharonov and L. Eldar, "On the complexity of commuting local Hamiltonians, and tight conditions for topological order in such systems," in 2011 IEEE 52nd Annual Symposium on Foundations of Computer Science (FOCS) (2011) pp. 334-343.

[11] Panos Aliferis and Andrew W. Cross, "Subsystem Fault Tolerance with the Bacon-Shor Code," Physical Review Letters 98, 220502 (2007).

[12] Andrew W. Cross, David P. Divincenzo, and Barbara M. Terhal, "A Comparative Code Study for Quantum Fault Tolerance," Quantum Info. Comput. 9, 541-572 (2009).

[13] Chip Elliott, "Building the quantum network," New Journal of Physics 4, 46 (2002).

[14] H. J. Kimble, "The quantum internet," Nature 453, 1023-1030 (2008).

[15] David Poulin, "Stabilizer Formalism for Operator Quantum Error Correction," Physical Review Letters 95, 230504 (2005).

[16] Courtney G. Brell, Steven T. Flammia, Stephen D. Bartlett, and Andrew C. Doherty, "Toric codes and quantum doubles from two-body Hamiltonians," New Journal of Physics 13, 053039 (2011).

[17] Julien Dorier, Federico Becca, and Frédéric Mila, "Quantum compass model on the square lattice," Physical Review B 72, 024448 (2005).

[18] A. J. Leggett, S. Chakravarty, A. T. Dorsey, Matthew P. A. Fisher, Anupam Garg, and W. Zwerger, "Dynamics of the dissipative two-state system," Reviews of Modern Physics 59, 1-85 (1987).

[19] Kristen L. Pudenz, Tameem Albash, and Daniel A. Lidar, "Error-corrected quantum annealing with hundreds of qubits," Nature Communications 5 (2014), $10.1038 /$ ncomms4243.

[20] Wojciech Brzezicki and Andrzej M. Oleś, "Symmetry properties and spectra of the twodimensional quantum compass model," Physical Review B 87, 214421 (2013).

\section{Appendix A: Rewriting the Hamiltonian in terms of auxiliary operators and stabilizers}

A critical step in calculating the energy separation for a two-local error-suppressing Hamiltonian arising from subsystem codes is rewriting the Hamiltonian in terms of auxiliary operators and stabilizers. This procedure makes explicit the dependence of the error-suppressing Hamiltonian on the values of the stabilizers. It also makes easier the analytical and numerical calculations of the energy separation by reducing the size of the Hilbert space that needs to be considered and removes the degeneracy in the error-suppressing Hamiltonian. Another application of such a procedure enables exact 
diagonalization of a $6 \times 6$ lattice of the quantum compass model [20].

Here, we describe a systematic method for finding the $m-k X$-type stabilizers, $m-k Z$-type stabilizers, and $n-2(m-k)-k X$-type auxiliary logical operators, and $n-2(m-k)-k Z$-type auxiliary logical operators, defining $n-2(m-k)-k$ auxiliary qubits for a $[[n, k, d]]$ generalized-Bacon-Shor code defined by a $m \times m$ binary matrix $M$ of rank $k$. We illustrate the application of this algorithm by using it to obtain a set of auxiliary operators and stabilizers for the $[[16,2,3]]$ generalized-Bacon-Shor code.

We first present pseudocode for the algorithm and then comment on its workings.

$\mathcal{A} \leftarrow \emptyset$

$\triangleright$ Holds set of auxiliary operators

$\mathcal{S} \leftarrow \emptyset$

$\triangleright$ Holds set of stabilizer generators

procedure Row EXTRACTION $(M)$

Let the set $\mathcal{R}$ hold all the rows of $M$

$\mathcal{R}_{\text {rem }} \leftarrow \mathcal{R}$

$\triangleright$ Holds remaining rows

$\mathcal{R}_{\text {cur }} \leftarrow \emptyset$

$\triangleright$ Holds rows under consideration

Add the top row of $M$ to $\mathcal{R}_{\text {cur }}$

while $\mathcal{R}_{\text {rem }}$ linearly dependent do

if $\mathcal{R}_{\text {cur }}$ linearly independent of $\mathcal{R}_{\text {rem }} \backslash \mathcal{R}_{\text {cur }}$ then

Move the rows in $\mathcal{R}_{\text {cur }}$ to the bottom of the matrix and set $\mathcal{R}_{\text {cur }} \leftarrow \emptyset$

Add the top row to $\mathcal{R}_{\text {cur }}$

end if

Add a minimally linearly independent set of rows in $\mathcal{R}_{\text {rem }} \backslash \mathcal{R}_{\text {cur }}$ to $\mathcal{R}_{\text {cur }}$ to make the top row linearly dependent on these rows and other rows in $\mathcal{R}_{\text {cur }}$

Move these rows to the top of the matrix, above rows already in $\mathcal{R}_{\text {cur }}$

Add to $\mathcal{S}$ the product of $Z$-type operators for every qubit in the rows of $\mathcal{R}_{\text {cur }}$

Add $X$-type operators to $\mathcal{A}$ corresponding to all pairs of qubits in the top row containing the first qubit

For all qubits in the top row except the first qubit, add $Z$-type operators to $\mathcal{A}$ that connect that qubit with the next qubit below it in its column

Remove the top row from $\mathcal{R}_{\text {cur }}$ and $\mathcal{R}_{\text {rem }}$

\section{end while}

Form $M^{\prime}$ from $\mathcal{R}_{\text {rem }}$

end procedure

procedure COLUMN EXTRACTION $\left(M^{\prime}\right)$

Let the set $\mathcal{C}^{\prime}$ hold all the columns of $M^{\prime}$

$\mathcal{C}_{\text {rem }} \leftarrow \mathcal{C}^{\prime}$

$\mathcal{C}_{\text {cur }} \leftarrow \emptyset$

Add the far left column of $M^{\prime}$ to $\mathcal{C}_{\text {cur }}$

while $\mathcal{C}_{\text {rem }}$ linearly dependent do

if $\mathcal{C}_{\text {cur }}$ linearly independent of $\mathcal{C}_{\text {rem }} \backslash \mathcal{C}_{\text {cur }}$ then

Move the columns in $\mathcal{C}_{\text {cur }}$ to the right side of the matrix and set

$\mathcal{C}_{\text {cur }} \leftarrow \emptyset$ 


\section{Add the far left column to $\mathcal{C}_{\text {cur }}$ end if}

Add a minimally linearly independent set of columns in $\mathcal{C}_{\text {rem }} \backslash \mathcal{C}_{\text {cur }}$ to $\mathcal{C}_{\text {cur }}$ to make it linearly dependent

Move these columns to the left side of the matrix

Add to $\mathcal{S}$ the product of $X$-type operators for every qubit in the corresponding columns to $\mathcal{C}_{\text {cur }}$ of the original matrix $M$

Add $Z$-type operators to $\mathcal{A}$ corresponding to all pairs of qubits in the left-most column containing the first qubit

for each qubit in the left-most column except the first qubit do in its row

Let $A_{X}$ be the $X$-type operator connecting that qubit with the next qubit

for $Z$-type operator $A_{Z}^{\prime}$ in $\mathcal{A}$ do

if $A_{X}$ anti-commutes with $A_{Z}^{\prime}$ then

Find the $X$-type operator $A_{X}^{\prime}$ that anticommutes with $A_{Z}^{\prime}$

$$
A_{X} \leftarrow A_{X} A_{X}^{\prime}
$$

end if

end for

end for

Remove the left-most column from $\mathcal{C}_{\text {cur }}$ and $\mathcal{C}_{\text {rem }}$

\section{end while}

Form $M^{\prime \prime}$ from $\mathcal{C}_{\text {rem }}$

\section{end procedure}

procedure CORE EXTRACTION $\left(M^{\prime \prime}\right)$

for each pair of adjacent qubits in same column of $M^{\prime \prime}$ do

Let $A_{Z}$ be the $Z$-type operator for this pair

for $X$-type operator $A_{X}^{\prime}$ in $\mathcal{A}$ do

if $A_{Z}$ anti-commutes with $A_{X}^{\prime}$ then

Find the $Z$-type operator $A_{Z}^{\prime}$ that anticommutes with $A_{X}^{\prime}$

$A_{Z} \leftarrow A_{Z} A_{Z}^{\prime}$

end if

\section{end for}

Add $A_{Z}$ to $\mathcal{A}$.

end for

for each adjacent pair of qubits in same row of $M^{\prime \prime}$ do

Let $A_{X}$ be the $X$-type operator for this pair

for $Z$-type operator $A_{Z}^{\prime}$ in $\mathcal{A}$ do

if $A_{X}$ anti-commutes with $A_{Z}^{\prime}$ then

Find the $X$-type operator $A_{X}^{\prime}$ that anticommutes with $A_{Z}^{\prime}$

$$
A_{X} \leftarrow A_{X} A_{X}^{\prime}
$$

end if

end for 


\section{Add $A_{X}$ to $\mathcal{A}$ \\ end for \\ end procedure}

Switching the order of the rows of a matrix defining a generalized-Bacon-Shor code does not change the gauge group, only the gauge generators. Moving rows added to $\mathcal{R}_{\text {cur }}$ to the top of the matrix ensures that the auxiliary $X$-type operators we define commute with all previously defined auxiliary operators. Specifically, the $Z$-type operators previously defined do not involve this row. The $Z$-type operators we define are all within $\mathcal{R}_{\text {cur }}$, because the constraint of minimal linear dependence guarantees that there will be a nonzero entry in the column below each qubit in the top row. We exclude the left-most column $Z$-type operator because it is the product of the stabilizer and the other $Z$-type operators which we define. Because we will not be including that column, we choose $X$-type operators that include the first qubit since that enables us to satisfy easily the canonical commutation relation for $X$ - and $Z$-type operators defining auxiliary qubits. Once we are considering columns, we must be more careful. While the $Z$-type operators we define automatically commute with all previously defined operators, because those rows were not used to extract $X$-type operators, this property is not guaranteed for the $X$-type operators, which is why the extraction of $X$-type operators at this state is more complicated than for $Z$-type operators.

\section{Example: Extracting auxiliary operators and stabilizers for the $[[16,2,3]]$ code}

The following symmetric matrix defines a generalized-Bacon-Shor [[16, 2, 3]] code,

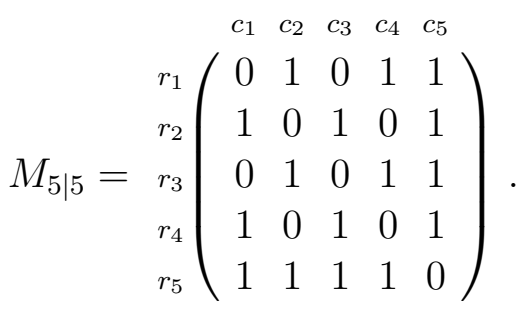

The subscripts in $M_{5 \mid 5}$ indicate the numbers of the rows and columns, respectively. We use $c_{j}$ and $r_{k}$ to label the row and column indices, so that we can keep track of the qubits after row and column manipulations. The rank of the matrix $M_{5 \mid 5}$ is 2 , and the distance of the code is 3 (since any linear combination of its rows or columns has either at least three $1 \mathrm{~s}$ or none). We will use the algorithm to find the $3 X$-type stabilizers, the $3 Z$-type stabilizers, and the 8 auxiliary qubits defined by 8 pairs of auxiliary operators satisfying the canonical commutation relations. 
Moving row $r_{3}$ to the top of the matrix yields

$$
M_{5 \mid 5}^{\prime}=\frac{\underline{r_{3}}}{\underline{r_{1}}}\left(\begin{array}{ccccc}
c_{1} & c_{2} & c_{3} & c_{4} & c_{5} \\
0 & 1 & 0 & 1 & 1 \\
r_{2} & 1 & 0 & 1 & 1 \\
r_{4} & 0 & 1 & 0 & 1 \\
r_{5} & 0 & 1 & 0 & 1 \\
1 & 1 & 1 & 1 & 0
\end{array}\right),
$$

where we underline the row labels to indicate the rows (columns) we are currently considering, rows that are in $\mathcal{R}_{c u r}$. Since the top two rows in $M_{5 \mid 5}^{\prime}$ are identical, and thus linearly dependent, we define a stabilizer $S_{1}^{Z}=R_{3}^{Z} R_{1}^{Z}$, where $R_{3}^{Z}=Z_{3,2} Z_{3,4} Z_{3,5}$ and $R_{1}^{Z}=Z_{1,2} Z_{1,4} Z_{1,5}$. We now extract auxiliary operators as we eliminate the top row. We define two auxiliary operators $X_{A 1}=X_{3,2} X_{3,4}$ and $X_{A 2}=X_{3,2} X_{3,5}$, and define the corresponding $Z$-type auxiliary operators to be $Z_{A 1}=Z_{3,4} Z_{1,4}$ and $Z_{A 2}=Z_{3,5} Z_{1,5}$. It is easy to check that two pairs of auxiliary operators satisfy the standard commutation relations. Note $Z_{3,2} Z_{1,2}=S_{1}^{Z} Z_{A 1} Z_{A 2}$. Having used the top row to obtain auxiliary operators and stabilizers, we may remove the top row. We consider the resulting matrix

$$
M_{4 \mid 5}=\begin{gathered}
r_{1} \\
r_{2} \\
r_{4} \\
r_{5}
\end{gathered}\left(\begin{array}{cccccc}
c_{1} & c_{2} & c_{3} & c_{4} & c_{5} \\
& 1 & 0 & 1 & 1 \\
1 & 0 & 1 & 0 & 1 \\
1 & 0 & 1 & 0 & 1 \\
1 & 1 & 1 & 1 & 0
\end{array}\right) .
$$

A minimally linearly independent set on which the top row is linearly dependent is $\left\{r_{2}, r_{5}\right\}$. We move these rows to the top of the matrix to obtain

$$
M_{4 \mid 5}^{\prime}=\frac{\underline{r_{2}}}{\underline{r_{5}}} \underset{\underline{r_{1}}}{\underline{r_{1}}}\left(\begin{array}{ccccc}
c_{4} & c_{2} & 1 & c_{4} & c_{5} \\
r_{4} & 1 & 1 & 1 & 0 \\
0 & 1 & 0 & 1 & 1 \\
1 & 0 & 1 & 0 & 1
\end{array}\right) .
$$

We define a stabilizer $S_{2}^{Z}=R_{2}^{Z} R_{5}^{Z} R_{1}^{Z}$, where $R_{2}^{Z}=Z_{2,1} Z_{2,3} Z_{2,5}, R_{5}^{Z}=Z_{5,1} Z_{5,2} Z_{5,3} Z_{5,4}$, and $R_{1}^{Z}=Z_{1,2} Z_{1,4} Z_{1,5}$. We define $X$-type auxiliary operators $X_{A 3}=X_{2,1} X_{2,3}$ and $X_{A 4}=X_{2,1} X_{2,5}$, and $Z$-type auxiliary operators $Z_{A 3}=Z_{2,3} Z_{5,3}$, and $Z_{A 4}=Z_{2,5} Z_{1,5}$. We may now remove the top row to obtain

$$
M_{3 \mid 5}=\frac{\underline{r_{5}}}{\underline{r}_{1}}\left(\begin{array}{ccccc}
c_{1} & c_{2} & c_{3} & c_{4} & c_{5} \\
r_{4} & 1 & 1 & 1 & 0 \\
0 & 1 & 0 & 1 & 1 \\
1 & 0 & 1 & 0 & 1
\end{array}\right) .
$$


We move $r_{4}$ to the top of the matrix to obtain

$$
M_{3 \mid 5}^{\prime}=\frac{\underline{r_{4}}}{\underline{r_{5}}}\left(\begin{array}{ccccc}
c_{1} & c_{2} & c_{3} & c_{4} & c_{5} \\
\underline{r_{1}} & 0 & 1 & 0 & 1 \\
1 & 1 & 1 & 1 & 0 \\
0 & 1 & 0 & 1 & 1
\end{array}\right) .
$$

We define a stabilizer $S_{3}^{Z}=R_{4}^{Z} R_{5}^{Z} R_{1}^{Z}$, where $R_{4}^{Z}=Z_{4,1} Z_{4,3} Z_{4,5}, R_{5}^{Z}=Z_{5,1} Z_{5,2} Z_{5,3} Z_{5,4}$, and $R_{1}^{Z}=Z_{1,2} Z_{1,4} Z_{1,5}$. We define $X$-type auxiliary operators $X_{A 5}=X_{4,1} X_{4,3}$ and $X_{A 6}=X_{4,1} X_{4,5}$, and $Z$-type operators $Z_{A 5}=Z_{4,3} Z_{5,3}$ and $Z_{A 6}=Z_{4,5} Z_{1,5}$. We may now remove the top row to obtain

$$
M_{2 \mid 5}={ }_{r_{1}}^{r_{5}}\left(\begin{array}{ccccc}
c_{1} & c_{2} & c_{3} & c_{4} & c_{5} \\
1 & 1 & 1 & 1 & 0 \\
0 & 1 & 0 & 1 & 1
\end{array}\right) .
$$

Now that the rows are linearly independent, we can engage "column elimination" to extract further operators. We move $c_{3}$ to the far left to obtain

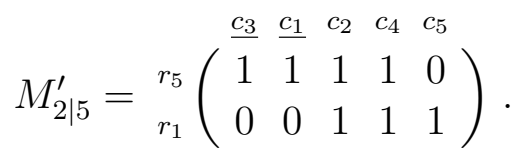

Were $M_{2 \mid 5}^{\prime}$ the starting matrix, the first $X$-type stabilizer would be $\tilde{S}_{1}^{X}=X_{5,3} X_{5,1}$. However, this $X$-type stabilizer does not commute with some of the $Z$-type auxiliary operators we introduced before. To obtain the correct stabilizer, we need to iteratively multiply $\tilde{S}_{1}^{X}$ by $X$-type operators corresponding to (anti-commuting with) $Z$-type operators that do not commute with $\tilde{S}_{1}^{X}$. It does not commute with $Z_{A 5}=Z_{4,3} Z_{5,3}$, so we need to multiply by $X_{A 5}=X_{4,1} X_{4,3}$, which in turn does not commute with $Z_{A 3}=Z_{2,3} Z_{5,3}$, so we need to multiply by $X_{A 3}=X_{2,1} X_{2,3}$. The result is the stabilizer

$$
\begin{aligned}
S_{1}^{X} & =X_{2,3} X_{4,3} X_{5,3} X_{2,1} X_{4,1} X_{5,1} \\
& =C_{3}^{X} C_{1}^{X} .
\end{aligned}
$$

The left-most column contains no pairs of $1 \mathrm{~s}$, so we do not extract any auxiliary operators at this step. We may now remove the far left column to obtain

$$
M_{2 \mid 4}={ }_{r_{1}}^{r_{5}}\left(\begin{array}{cccc}
\frac{c_{1}}{1} & c_{2} & c_{4} & c_{5} \\
1 & 1 & 1 & 0 \\
0 & 1 & 1 & 1
\end{array}\right) .
$$

Columns $c_{2}$ and $c_{5}$ form a minimally linearly independent set on which the left-most column $c_{1}$ depends. We therefore move these columns to the far left to obtain

$$
M_{2 \mid 4}^{\prime}={ }_{r_{1}}^{r_{5}}\left(\begin{array}{cccc}
\frac{c_{2}}{1} & \frac{c_{5}}{0} & \frac{c_{1}}{1} & c_{4} \\
1 & 1 & 0 & 1
\end{array}\right) .
$$


A similar argument to the one above leads to defining the stabilizer

$$
\begin{aligned}
S_{2}^{X} & =X_{1,2} X_{3,2} X_{5,2} X_{1,5} X_{2,5} X_{3,5} X_{4,5} X_{2,1} X_{4,1} X_{5,1} \\
& =C_{2}^{X} C_{5}^{X} C_{1}^{X} .
\end{aligned}
$$

We also define the pair of operators $Z_{A 7}=Z_{5,2} Z_{1,2}$ and $\tilde{X}_{A 7}=X_{1,2} X_{1,5}$. Since $\tilde{X}_{A 7}$ anticommutes with $Z_{A 2}, Z_{A 4}$, and $Z_{A 6}$, we have $X_{A 7}=\tilde{X}_{A 7} X_{A 2} X_{A 4} X_{A 6}$. We now remove the left-most column to obtain

$$
M_{2 \mid 3}={ }_{r_{1}}^{r_{5}}\left(\begin{array}{lll}
\frac{c_{5}}{0} & \frac{c_{1}}{1} & c_{4} \\
1 & 0 & 1
\end{array}\right) .
$$

Moving the row $c_{4}$ to far left, we have

$$
M_{2 \mid 3}^{\prime}={ }_{r_{1}}^{r_{5}}\left(\begin{array}{lll}
\frac{c_{4}}{1} & \frac{c_{5}}{0} & \frac{c_{1}}{1} \\
1 & 1 & 0
\end{array}\right) .
$$

These columns contribute a stabilizer

$$
\begin{aligned}
S_{3}^{X} & =C_{4}^{X} C_{5}^{X} C_{1}^{X} \\
& =X_{1,4} X_{3,4} X_{5,4} X_{1,5} X_{2,5} X_{3,5} X_{4,5} X_{2,1} X_{4,1} X_{5,1} .
\end{aligned}
$$

We also extract the final set of auxiliary operators $Z_{A 8}=Z_{5,4} Z_{1,4}$ and $X_{A 8}=$ $\tilde{X}_{A 8} X_{A 1} X_{A 2} X_{A 4} X_{A 6}$, where $\tilde{X}_{A 8}=X_{1,4} X_{1,5}$. Removing the first column results in a matrix with linearly independent rows and columns and in which no row or column contains a pair of $1 \mathrm{~s}$; therefore we stop extracting operators. Indeed, we have already obtained $3 X$-type and $3 Z$-type stabilizers and 8 pairs of auxiliary operators as expected.

In conclusion, we have following auxiliary operators

$$
\begin{gathered}
X_{A 1}=X_{3,2} X_{3,4}, \quad X_{A 2}=X_{3,2} X_{3,5}, \quad X_{A 3}=X_{2,1} X_{2,3}, \\
X_{A 4}=X_{2,1} X_{2,5}, \quad X_{A 5}=X_{4,1} X_{4,3}, \quad Z_{A 1}=Z_{1,4} Z_{3,4}, \\
Z_{A 2}=Z_{1,5} Z_{3,5}, \quad Z_{A 3}=Z_{2,3} Z_{5,3}, \quad Z_{A 4}=Z_{2,5} Z_{1,5} \\
Z_{A 5}=Z_{4,3} Z_{5,3}, \quad X_{A 6}=X_{4,1} X_{4,5}, \quad X_{A 7}=X_{1,2} X_{1,5} X_{A 2} X_{A 4} X_{A 6}, \\
X_{A 8}=X_{1,4} X_{1,5} X_{A 1} X_{A 2} X_{A 4} X_{A 6}, \quad Z_{A 6}=Z_{4,5} Z_{1,5}, \\
Z_{A 7}=Z_{5,2} Z_{1,2}, \quad Z_{A 8}=Z_{5,4} Z_{1,4},
\end{gathered}
$$

and the stabilizers take the form

$$
\begin{aligned}
& S_{1}^{X}=C_{3}^{X} C_{1}^{X}, \quad S_{2}^{X}=C_{2}^{X} C_{5}^{X} C_{1}^{X}, \quad S_{3}^{X}=C_{5}^{X} C_{1}^{X} C_{4}^{X}, \\
& S_{1}^{Z}=R_{1}^{Z} R_{3}^{Z}, \quad S_{2}^{Z}=R_{2}^{Z} R_{5}^{Z} R_{1}^{Z}, \quad S_{3}^{Z}=R_{5}^{Z} R_{1}^{Z} R_{4}^{Z} .
\end{aligned}
$$




\section{Appendix B: Noise model}

For our numerical analyses of error suppression, we consider the spin-boson Hamiltonian [18],

$$
H(t)=H_{S}(t)+H_{B}+\sum_{k=1}^{n}\left(X_{k} \otimes B_{k}^{X}+Y_{k} \otimes B_{k}^{Y}+Z_{k} \otimes B_{k}^{Z}\right),
$$

where $H_{S}=H_{\text {supp }}+H_{L}$ is the system Hamiltonian, and $X_{k}, Y_{k}$, and $Z_{k}$ are Pauli operators acting on the $k$ th qubit. The sum in Eq. (B1) describes interactions between individual Pauli operators of the system qubits and independent bath modes, where

$$
\begin{aligned}
B_{k}^{X} & =\sum_{\mu} g_{\mu}^{X}\left(a_{\mu, k}+a_{\mu, k}^{\dagger}\right), \\
B_{k}^{Y} & =\sum_{\nu} g_{\nu}^{Y}\left(b_{\nu, k}+b_{\nu, k}^{\dagger}\right) \\
B_{k}^{Z} & =\sum_{\tau} g_{\tau}^{Z}\left(c_{\tau, k}+c_{\tau, k}^{\dagger}\right),
\end{aligned}
$$

with $g_{\mu}^{X}, g_{\nu}^{Y}$, and $g_{\tau}^{Z}$ being the coupling constants. We consider the case in which all of these coupling constants have the same value. The term $H_{B}$ in Eq. (B1) is the bath Hamiltonian,

$$
H_{B}=\sum_{\mu, j} \hbar \omega_{\mu, j}^{X} a_{\mu, j}^{\dagger} a_{\mu, j}+\sum_{\nu, k} \hbar \omega_{\nu, k}^{Y} b_{\nu, k}^{\dagger} b_{\nu, k}+\sum_{\tau, l} \hbar \omega_{\tau, l}^{Z} c_{\tau, l}^{\dagger} c_{\tau, l} .
$$

Going to the Heisenberg picture of the bath Hamiltonian, we have

$$
B_{k}(t)=e^{i t H_{B} / \hbar} B_{k} e^{-i t H_{B} / \hbar}=\sum_{\mu}\left(g_{\mu} a_{\mu, k} e^{-i \omega_{\mu} t}+g_{\mu}^{*} a_{\mu, k}^{\dagger} e^{i \omega_{\mu} t}\right)
$$

where the superscripts $X, Y$, and $Z$ are neglected for abbreviation of notation. The bath correlation function then takes the form

$$
\begin{aligned}
C_{\mathrm{bath}}\left(j, t ; k, t^{\prime}\right) & =\left\langle B_{j}(t) B_{k}\left(t^{\prime}\right)\right\rangle \\
& =\delta_{j, k} \sum_{\mu}\left|g_{\mu}\right|^{2}\left(\left\langle a_{\mu, k}^{\dagger} a_{\mu, k}\right\rangle e^{-i \omega_{\mu, k}\left(t-t^{\prime}\right)}+\text { c.c. }\right) \\
& =\delta_{j, k} C_{\mathrm{bath}}\left(t-t^{\prime}\right)
\end{aligned}
$$

The expectation values in Eq. (B7) satisfy the Planck condition for thermal baths,

$$
\left\langle a_{\mu}^{\dagger} a_{\mu}\right\rangle=\frac{1}{e^{\hbar \omega_{\mu} / k_{\mathrm{B}} T}-1}, \quad\left\langle a_{\mu} a_{\mu}^{\dagger}\right\rangle=\left\langle a_{\mu}^{\dagger} a_{\mu}\right\rangle+1=\frac{1}{1-e^{-\hbar \omega_{\mu} / k_{\mathrm{B}} T}},
$$


where the qubit subscript $k$ is omitted. The Fourier transformation of the bath correlation function is

$$
\widetilde{C}_{\text {bath }}(\omega)=\int d t e^{-i \omega t} C_{\text {bath }}(t)=\frac{2 \pi J(|\omega|)}{\mid 1-e^{-\hbar \omega / k_{\mathrm{B}} T \mid}},
$$

where $J(\omega)$ is the bath spectral function arising from the substitution of the sum in Eq. (B7) with an integral,

$$
\sum_{\mu}\left|g_{\mu}\right|^{2} \simeq \int_{0}^{\infty} d \omega J(\omega) .
$$

The bath correlation function determines the transition rate from one system state $\left|\psi_{\alpha}\right\rangle$ to another state $\left|\psi_{\beta}\right\rangle$, where $E_{\alpha}$ and $E_{\beta}$ are the energies of the two states and $Q$ is the system noise operator (in this case $X, Y$, and $Z$ ). The ratio of the transition rates between any two states satisfies

$$
\frac{\Gamma_{\psi_{\alpha} \rightarrow \psi_{\beta}}}{\Gamma_{\psi_{\beta} \rightarrow \psi_{\alpha}}}=e^{\left(E_{\alpha}-E_{\beta}\right) / k_{\mathrm{B}} T},
$$

which gives the correct population ratio of $\left|\psi_{\beta}\right\rangle\left\langle\psi_{\beta}\right|$ and $\left|\psi_{\alpha}\right\rangle\left\langle\psi_{\alpha}\right|$ at thermal equilibrium, i.e., the Boltzmann distribution. The function $\widetilde{C}_{\text {bath }}(\omega)$ determines the transition rate from a lower-energy state to a higher-energy state when $\omega<0$, and the other way around when $\omega>0$. While transitions to higher-energy states are detrimental, transitions to lower-energy states are beneficial for adiabatic quantum computation.

We further assume that the bath spectral function satisfies the Ohmic condition,

$$
J(\omega) \simeq \hbar^{2} \chi \omega e^{-\omega / \omega_{c}}, \quad \text { for } \omega \geq 0,
$$

where $\chi$ is a dimensionless constant and $\omega_{c}$ is the cutoff frequency. The bath correlation function can thus be simplified to

$$
\widetilde{C}_{\text {bath }}(\omega)=\frac{2 \pi \hbar^{2} \chi \omega e^{-|\omega| / \omega_{c}}}{1-e^{-\omega / \omega_{T}}},
$$

where $\omega_{T}=k_{\mathrm{B}} T / \hbar$. We plot this function with the parameters given in [19]: $\chi=$ $3.18 \times 10^{-4}, \omega_{c}=8 \pi \times 10^{9} \mathrm{rad} / \mathrm{s}$, and $\omega_{T}=2.2 \times 10^{9} \mathrm{rad} / \mathrm{s}($ at $17 \mathrm{mK})$. At zero frequency, i.e., transitions between two states of the same energy, $\widetilde{C}_{\text {bath }}(0)=2 \pi \hbar^{2} \chi \omega_{T}$ is proportional to the temperature $T$. The derivative of $\widetilde{C}_{\text {bath }}(\omega)$ is not continuous at $\omega=0$ due to the finite cutoff frequency $\omega_{c}$. The function $\widetilde{C}_{\text {bath }}(\omega)$ decays quickly once $\omega$ is smaller than $-\omega_{T}$; the transition rate to higher-energy states is low for an energy difference that is several times larger than $k_{\mathrm{B}} T$. Consequently, an energy gap as large as several times of $k_{\mathrm{B}} T$ can keep the system in the ground state for a much longer time than the gapless case. The asymmetry of the function $\widetilde{C}_{\text {bath }}(\omega)$ (see Figure 6) can also be used to prepare the initial state when the energy gap is less than $\hbar \omega_{c}$ but larger than $\hbar \omega_{T}$, as the noise terms drive the system to its ground state while the opposite effect is suppressed. 


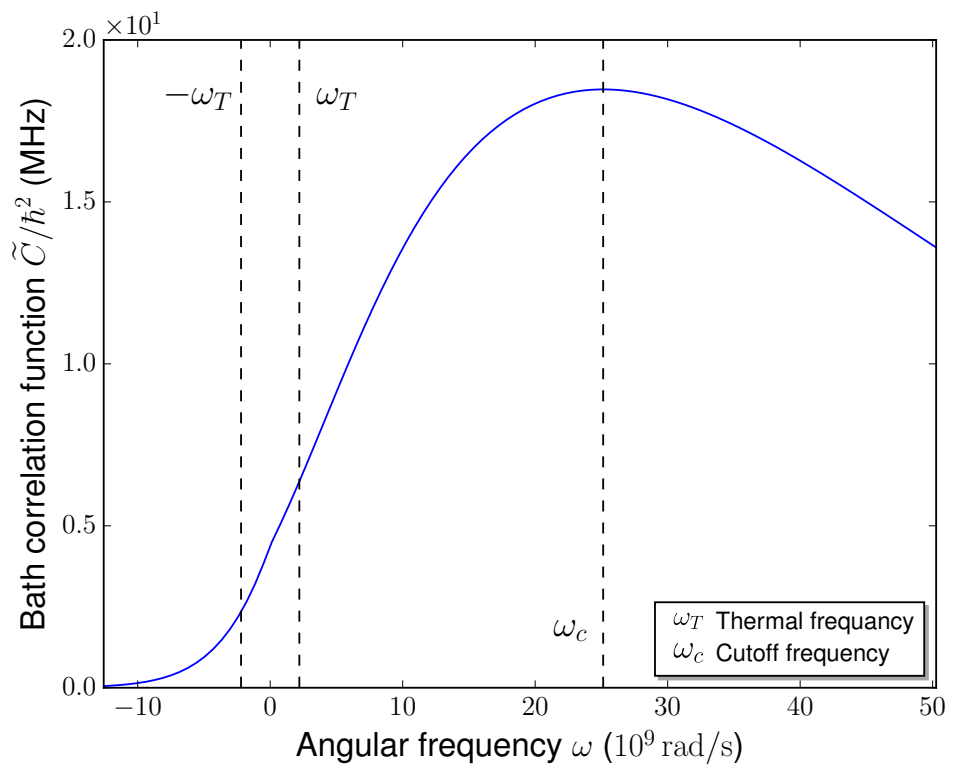

FIG. 6. Bath correlation function for Ohmic noise 